\section{$\underset{\substack{\text { hommes } \\ \text { \& migrations }}}{ }$}

\section{Hommes \& migrations}

Revue française de référence sur les dynamiques

migratoires

$1283 \mid 2010$

Cuisines et dépendances

\title{
La construction sociale de la cuisine péruvienne
}

Une histoire de migrations et d'échanges culinaires

\section{Raúl Matta}

\section{(2) OpenEdition}

12 Journals

\section{Édition électronique}

URL : http://journals.openedition.org/hommesmigrations/996

DOI : 10.4000/hommesmigrations.996

ISSN : 2262-3353

Éditeur

Musée national de l'histoire de l'immigration

\section{Édition imprimée}

Date de publication : 1 janvier 2010

Pagination : 96-107

ISBN : 978-2-919040-04-9

ISSN : $1142-852 X$

\section{Référence électronique}

Raúl Matta, «La construction sociale de la cuisine péruvienne », Hommes \& migrations [En ligne], 1283 | 2010, mis en ligne le 29 mai 2013, consulté le 19 avril 2019. URL : http://

journals.openedition.org/hommesmigrations/996 ; DOI : 10.4000/hommesmigrations.996 


\section{La construction sociale de la cuisine péruvienne Une histoire de migrations et d'échanges culinaires}

Par Raúl Matta, docteur en sociologie à l'université Paris 3 - Sorbonne Nouvelle/lHEAL, chercheur associé au CREDAL (Centre de recherche et de documentation sur l'Amérique latine)

Le Pérou vit actuellement un engouement sans précédent autour de sa cuisine, au niveau local comme à l'international. Ce véritable boom aux allures de révolution gastronomique est rendu possible par la richesse du patrimoine culinaire péruvien. Les phénomènes migratoires ont joué un rôle fondamental dans la construction de cette expression culturelle qui repose sur un mariage inédit des influences andines, italiennes, chinoises ou japonaises. À Lima, des chefs réputés travaillent à renouveler en profondeur ce métissage. 
Durant les cinq dernières années, les Péruviens n'ont jamais autant parlé de cuisine, de leurs chefs cuisiniers - considérés comme des véritables artistes derrière et devant les fourneaux -, de la qualité de la gastronomie péruvienne et de son grand potentiel d'internationalisation ${ }^{(1)}$. Cet intérêt généralisé pour la gastronomie a donné lieu à un certain nombre d'études qui dressent le constat suivant : cette cuisine tant célébrée est le fruit de plus de cinq siècles d'échanges culturels, des échanges qui se poursuivent jusqu'à nos jours.

Penser la cuisine péruvienne en fonction de la notion de métissage ou de brassage culturel $^{(2)}$ implique de retracer les événements historiques qui ont mis en place des passerelles entre les différentes cultures et façonné cette "cuisine métisse". Trois phénomènes migratoires - très distincts et chronologiquement éloignés les uns des autres - ont joué un rôle fondamental dans la construction de cette expression culturelle. Le premier, évoqué précédemment, remonte au temps des grandes découvertes et, bien qu'il ne s'agisse pas d'un phénomène concernant uniquement le Pérou, il a produit ses propres spécificités dans le domaine de l'alimentation. Une deuxième grande vague migratoire eut lieu entre la deuxième moitié du XIX ${ }^{\mathrm{e}}$ siècle et la première moitié du $\mathrm{XX}^{\mathrm{e}}$ siècle. Durant cette période débar-

Durant les cinq dernières années, les Péruviens n'ont jamais autant parlé de cuisine, de leurs chefs cuisiniers, de la qualité de la gastronomie péruvienne et de son grand potentiel d'internationalisation. quèrent sur la côte péruvienne des immigrants italiens - des commerçants cherchant à faire fortune -, des immigrants chinois et des immigrants japonais - des esclaves agricoles -, populations ayant laissé des empreintes culinaires ineffaçables. Dans ce cas, la migration humaine s'est vue accompagnée d'une migration de denrées alimentaires et d'un développement commercial lié à la présence de migrants, processus susceptibles d'élargir le spectre culinaire au sein de la société d'accueil.

Le résultat de ces deux héritages est la cuisine criolla, ou cuisine créole péruvienne. Cuisine métisse, côtière et urbaine, elle constitue le véritable fer de lance du renouveau gastronomique péruvien, dans la mesure où, en raison de ses caractéristiques assimilatrices, elle a préparé le terrain pour de nouvelles rencontres culinaires, en particulier celles résultant d'un flux migratoire d'une autre nature et, d'ailleurs, moins étudié : une "migration d'élite". Moins important en nombre, ce troisième type de migration fut cependant essentiel à la valorisation des caractéristiques culinaires autochtones parmi les "grandes tables" liméniennes et au récent rayonnement international de la gastronomie péruvienne. 


\section{Quand la cuisine des vainqueurs rencontre celle des vaincus}

Vers 1554, après le massacre des autochtones lors de la guerre de conquête et les guerres intestines dans le camp des conquistadors, les Espagnols qui y ont survécu vivaient comme de grands seigneurs. En effet, ils avaient réussi à partager le butin - l'or et l'argent des mines -, ainsi que les terres agricoles et la main-d'ceuvre, grâce à la création des encomiendas ou "répartitions d'indiens", véritables unités féodales attribuées aux colons en fonction de leur apport à l'entreprise de conquête et à leur mérite en tant que guerriers.

Ces aventuriers avaient réalisé en quelque sorte leur rêve de vivre dans l'opulence, malgré la distance qui les séparait de leur pays d'origine. Soucieux de préserver un régime alimentaire espagnol, ils importèrent cependant de moins en moins de produits comestibles, car, en raison des techniques d'élevage et de l'agriculture intensive appliquée lors de leur établissement sur le sol américain, il était désormais possible de trouver un grand nombre de produits européens dans la production locale ${ }^{(3)}$.

Ainsi, les Espagnols anoblis et enrichis ne mangeaient plus de piment, ni de légumes, ni de tubercules déshydratés - produits qu'ils ont été obligés de consommer durant les guerres et les expéditions dans le but de survivre ${ }^{(4)}-$, aliments perçus comme étant indignes d'une grande table. Pourtant, leur cuisine contenait déjà des traces de produits américains, à la différence que ceux-ci étaient cuisinés à l'usage européen par les cuisinières morisques ${ }^{(5)}$ qu'ils emmenèrent dans leurs expéditions. En effet, il est important de signaler que les conquistadors sont arrivés au Pérou presque quarante ans après la découverte de l'Amérique, ce qui implique que le personnel au service des Espagnols était déjà familiarisé avec certains ingrédients du nouveau continent apportés par les colons lors de leurs retours en Europe ${ }^{(6)}$. Par exemple, l'historien Juan José Vega rapporte que les cuisinières morisques auraient introduit l'utilisation du riz comme garniture principale de la cuisine de la conquête en remplacement de la semoule, introuvable sur les terres américaines ${ }^{(7)}$.

Lors des banquets offerts par cette noblesse militaire, les viandes de tous types étaient omniprésentes et, éventuellement, se "glissaient", en guise d'accompagnement, au milieu de produits autochtones tels qu'une patate douce braisée, un épi de maïs ou une pointe de piment pour relever encore plus le goût des plats. En somme, et malgré la présence aléatoire d'ingrédients indigènes, la cuisine des conquistadors fortunés essayait de s'apparenter le plus possible à celle de la fin du Bas Moyen Âge européen et des débuts de la Renaissance. 


\section{Naissance de la cuisine criolla}

Au-delà des pratiques ostentatoires des vainqueurs, l'organisation politique du viceroyaume a eu d'importantes conséquences sur les pratiques alimentaires et culinaires de la société dans son ensemble. En effet, après les encomiendas, les Espagnols ont imposé une nouvelle structuration de la société indienne dans le but de mieux les contrôler et de mieux gérer la collecte des taxes. Cette institution coloniale mise en place en 1571, qui a pris le nom de "reducción”, a obligé les Indiens à se rassembler dans les villages situés dans les vallées andines. Elle signait ainsi l'acte de décès de l'agriculture traditionnelle en raison de l'abandon des terres d'altitude ${ }^{(8)}$. Parmi la nouvelle production agricole se trouvaient aussi bien des produits d'origine européenne - qui poussaient sans aucun problème sur les terres du nouveau continent - que des produits locaux, ces derniers étant principalement utilisés pour nourrir les domestiques et les esclaves agricoles. De ce fait, les aliments américains et européens ont fini par se côtoyer dans les celliers et dans les cuisines des demeures seigneuriales, mais en menant des vies parallèles. Cependant, cette séparation ne pouvait résister à la coexistence de deux cultures sur un même territoire. Selon Hinostroza, trois principes fondamentaux sont à l'origine des nombreuses transformations culinaires ayant eu lieu à partir de ce moment ${ }^{(9)}$.

Le premier consiste à substituer un élément à un autre similaire - par exemple, du blé remplaçant le maïs ou

Parmi la nouvelle production agricole se trouvaient aussi bien des produits d'origine européenne que des produits locaux, ces derniers étant principalement utilisés pour nourrir les domestiques et les esclaves agricoles. l'utilisation de pâtes au lieu de riz. Cette opération est courante en temps de carence, mais parfois elle est capable de transformer un plat en un autre très différent, en particulier si ce qui est remplacé est l'ingrédient principal.

Le deuxième principe consiste à appliquer une technique culinaire connue à un ingrédient inconnu. Tel est le cas de la farce à base de viande hachée qui, en Europe, était utilisée pour remplir les volailles. Cette technique, appliquée à des produits comme la pomme de terre (papa rellena) ou à certains piments (rocoto relleno), offre un résultat tout à fait original. Une autre variante de cette technique consiste à ajouter des ingrédients à un plat qui ne les contient pas habituellement, par exemple le fait de rajouter un ceuf et du lait - ingrédients interdits dans la cuisine inca - à la base autochtone de la soupe aux crevettes (crevettes, pommes de terre, piment), créant ainsi l'actuelle chupe de camarones. 
Le troisième principe consiste à introduire de nouveaux assaisonnements dans les plats comme, par exemple, l'ajout de piment et d'herbe huacatay dans un sancochado - équivalent péruvien du cocido madrileño (viandes et légumes cuits à la vapeur). Ces techniques, appliquées à l'importante variété de produits existant sur les terres coloniales, ont été sans aucun doute à l'origine du métissage culinaire péruvien ou, plus précisément, d'une "troisième cuisine": la cuisine criolla ${ }^{(10)}$. Développée à Lima, capitale du vice-royaume du Pérou, cette expression culinaire illustre la rencontre de cultures et les échanges subséquents à cette époque.

\section{Les migrations du $\mathrm{XIX}$ siècle et l'élargissement du répertoire culinaire péruvien}

Le 28 juillet 1821, José de San Martin déclarait l'indépendance du Pérou à la suite d'une série de "guerres d'indépendance" menées par l'élite criolla hispanoaméricaine, lassée de rendre des comptes à la couronne espagnole et d'être exclue de la vie politique.

Une des premières mesures adoptées par le nouveau gouvernement consistait à expulser les Espagnols du territoire péruvien. Seuls étaient tolérés les Espagnols pacifiques et respectueux des lois de la République. Ainsi, les autorités nommées par la couronne, les militaires, les gendarmes, et un bon nombre de riches commerçants s'embarquèrent pour la péninsule Ibérique. Cette mesure radicale libéra les enclaves commerciales détenues par la descendance des premiers colons, désormais prêtes à accueillir de nouveaux capitaux. Le contexte économique des débuts de la République fut alors marqué par l'entrée de produits divers - liqueurs, vêtements, tissus, aliments, outils et matériel de bricolage - en provenance d'Angleterre, des États-Unis, d'Italie et de France, en remplacement de ceux venus d'Espagne ${ }^{(11)}$.

En matière culinaire, la cuisine française occupa la place laissée par la cuisine espagnole, devenant ainsi synonyme d'élégance, de culture et de savoir-faire aux yeux des catégories aisées. Néanmoins, et en raison de sa forte inscription au sommet de la hiérarchie sociale, la cuisine française n'a pas influencé la cuisine criolla - qui, elle, incorporait aussi bien quelques éléments des "grandes tables" que certains éléments des "tables populaires". Elle n’a pas non plus encouragé de techniques de substitution d'ingrédients ni élargi le régime alimentaire du plus grand nombre, à la différence des apports spécifiques des migrants chinois, italiens et japonais, arrivés - dans cet ordre chronologique - au cours du XIX ${ }^{\mathrm{e}}$ siècle. 


\section{La marque chinoise : des plats copieux et populaires}

Linfluence culinaire chinoise au Pérou est d'une nature semblable à l'influence culinaire française, dans la mesure où la cuisine chinoise et la cuisine criolla n'ont pas produit des formes culinaires "métisses", à l'exception du lomo saltado (émincé d'échine de bceuf sauté aux oignons, servi avec des frites et du riz blanc), dont la préparation a introduit la technique du "sauté" dans l'inventaire des pratiques culinaires péruviennes. La reproduction des pratiques culinaires chinoises dans le pays d'accueil a cependant contribué à l'élargissement du régime alimentaire péruvien. En effet, la cuisine développée par la progéniture des travailleurs agricoles arrivés de Guangzhou (Canton) à partir de la deuxième moitié du XIX siècle a toujours gardé son essence cantonaise. D'ailleurs, il semblerait que l'un des facteurs clés de l'intégration des Chinois à la société péruvienne ait été la préservation de leur régime alimentaire : les contrats qui liaient ces immigrants aux hacendados (propriétaires terriens) contenaient une clause précisant que chaque Chinois devait recevoir une certaine quantité de riz, de viande salée et de légumes nécessaires à son alimentation $^{(12)}$.

Une fois leurs contrats arrivés à terme et non renouvelés en raison de l'essor d'un courant abolitionniste opposé aux dures conditions de travail dans les haciendas, les Chinois et leurs descendants se sont installés principalement tout du long de la côte péruvienne - bien qu'ils aient aussi peuplé l'Amazonie et, dans une moindre mesure, les Andes ${ }^{(13)}$. Ils firent de la restauration et du petit commerce leurs principales activités de subsistance. Dans les fondas ${ }^{(14)}$, ils proposaient des plats simples et bon marché très bien accueillis par les classes populaires. Plus tard, leur activité dans la restauration prit plus d'envergure, donnant ainsi naissance aux chifas ${ }^{(15)}$, des restaurants spécialisés dans la cuisine chinoise. Ces établissements ont gagné en visibilité à partir des années vingt, lorsque les classes supérieures liméniennes furent attirées par le cadre luxueux et la variété de l'offre gastronomique qui leur était proposés - ce qui contribua aussi à atténuer les préjugés et le racisme envers cette communauté.

Toutefois, l'influence de la cuisine chinoise se consolida davantage dans des milieux plus modestes, le terme chifa évoquant actuellement une cuisine populaire copieusement servie dans des restaurants où le temps d'attente est réduit et dont les cartes de menu montrent des photos de "formules" proposées à un prix assez abordable. Le chifa est ainsi devenu - à quelques exceptions près ${ }^{(16)}$ - un type de restauration proche du fast-food nord-américain, tandis que les restaurants de cuisine chinoise haut de gamme préfèrent se positionner en tant que "restaurants 
orientaux". La diffusion de cette cuisine parmi l'ensemble des classes sociales a donc influencé fortement les habitudes alimentaires des Péruviens, désormais grands consommateurs de riz, et donné lieu à une production agricole d'origine chinoise sur le sol péruvien. Ainsi, un des plats de base du chifa, l'arroz chaufa (riz cantonais sauté), a fini par intégrer le répertoire culinaire criollo ; il est parfois servi en tant qu'accompagnement, en remplacement du riz blanc.

\section{Des pâtes au pannetone, l'apport des migrants italiens}

La migration italienne vers le Pérou, dont les débuts se situent au cours des années dix-huit cent soixante-dix, fut faible par rapport à celle connue par l'Argentine à la même époque - un peu plus de 10000 migrants ont débarqué sur les côtes péruviennes contre 2 millions sur les côtes argentines ${ }^{(17)}$. Pourtant, cela n'a pas empêché l'hégémonie de cette colonie dans le secteur du petit commerce liménien grâce à leurs épiceries, bazars, cafés, boulangeries et pulperias ${ }^{(18)}$. Par la suite, les Italiens furent reconnus comme d'habiles entrepreneurs, compte tenu de leur incursion dans des domaines assez dissemblables, tels que la banque, l'industrie textile, l'hôtellerie et surtout l'industrie alimentaire, dans laquelle ils excellèrent. En effet, ils montèrent des usines de fabrication de pâtes, des chocolateries, des gelaterias, des usines de panification, des usines de production d'huile comestible,

\section{Le minestrone, soupe traditionnelle italienne, se transforme en menestrón Iorsqu'on lui ajoute des haricots et du maïs.} entre autres.

Ces bases solides ont permis à l'influence culinaire italienne de ne pas se limiter à une simple question de mode - comme ce fut le cas de la cuisine française - mais, au contraire, d'être soutenue par une prospère industrie alimentaire. Ainsi, de nos jours, manger le pannetone à Noël est une véritable tradition partagée par toutes les classes sociales. Il est aussi très apprécié au cours de la première grande fête de l'année, la fête nationale péruvienne. De même, les pâtes sont devenues un produit indispensable dans le régime alimentaire des Péruviens. Peu coûteuses, faciles à transporter et à emmagasiner, elles sont aussi consommées dans les zones les plus reculées du pays.

En ce qui concerne la préparation des aliments, nous pouvons identifier quelques exemples de métissage italo-péruvien, telles que les spaghettis à la sauce huancaina - sauce d'origine andine à base de fromage frais et de piment jaune -, un plat de 
grande diffusion sur les tables populaires, ou les tallarines en salsa verde, une version péruvienne des spaghettis à la sauce pesto. Si la recette italienne de cette sauce contient du basilic, des pignons de pin, de l'huile d'olive et du fromage parmesan, la recette de la "sauce verte" péruvienne rajoute des épinards et remplace le parmesan par du fromage frais sans sel et les pignons de pin par des noix. Mais l'influence italienne ne se résume pas à la consommation de pâtes. Le minestrone, soupe traditionnelle italienne, se transforme en menestrón lorsqu'on lui ajoute des haricots et du maïs. Aussi, dans la plupart des restaurants situés sur le littoral péruvien, on trouve un plat appelé "conchitas a la parmesana" (coquilles SaintJacques gratinées au parmesan), plat qui a disparu dans les restaurants de la région lombarde d'Émilie-Romagne (villes de Parme, Bologne, Ferrare).

\section{Le cebiche à la mode japonaise ou la victoire du poisson cru}

Les premiers immigrants japonais arrivèrent au Pérou en 1899. Comme dans le cas des Chinois, il s'agissait de paysans pauvres venus pour travailler sur les haciendas de la côte dans des conditions esclavagistes. Lors de leur arrivée, ils s'installèrent eux aussi dans la capitale et les villes côtières. Les Japonais ont concurrencé avec succès les commerces des premiers immigrants asiatiques, car ils étaient considérés par les Liméniens "de souche" comme des individus propres - réputation déniée aux Chinois sur le plan alimentaire -, honnêtes et comme des commerçants sérieux et bon marché.

Parmi leurs petites entreprises, celles liées à la restauration ont fortement influencé les habitudes alimentaires liméniennes, notamment en s'attaquant au plat emblématique de la cuisine péruvienne et de l'individu criollo : le cebiche ${ }^{(19)}$. Plus tard, au cours des années soixante-dix, les restaurateurs nisei ${ }^{(20)}$ ont mis à profit le large succès de ce plat, en généralisant sa présence chez les petits aubergistes. Mais leur préparation du cebiche différait de celle qui avait été popularisée quelques décennies auparavant. Dans leur version, l'influence japonaise était évidente, car le poisson était servi pratiquement cru, presque comme du sashimi. De nos jours, cette recette du cebiche est considérée par les critiques gastronomiques péruviens comme étant plus savoureuse et raffinée que la recette traditionnelle, qui exigeait le marinage du poisson dans le jus de citron pendant des heures, afin qu'il ne soit pas servi cru. Aujourd'hui, le cebiche ancien, de texture caoutchouteuse et avec les oignons cuits par l'acidité du citron, est devenu obsolète et souvent considéré comme immangeable sur certaines tables liméniennes ${ }^{(21)}$. 
La découverte réalisée par les nisei a donné lieu à la multiplication des dénommées "cebicherias" dans toute la capitale. À partir des années quatre-vingt, les premiers restaurants de cuisine nikkei - terme qui signifie d'"origine japonaise" en référence aux immigrants japonais et à leur descendance ${ }^{(22)}$ - voient le jour. Le métissage entre la cuisine criolla et la cuisine japonaise s'est poursuivi dans ces lieux, proposant une alliance entre les sauces d'origine péruvienne l'utilisation de sauces dans la cuisine japonaise est très réduite - et la rigueur technique japonaise appliquée au traitement du poisson et de fruits de mer frais. Cette alliance a donné lieu à une variété de plats inédits : escargots de mer à la sauce soja, pulpo al olivo (poulpe servi avec une mayonnaise faite à base d'olives noires péruviennes), cebiche à la sauce soja et à la moutarde, poisson farci aux fruits de mer, calamars farcis au saumon.

\section{Le retour des "migrants d'élite" et l'avènement d'un nouveau métissage culinaire}

Suivant les tendances internationales et maitrisant les aspects techniques et discursifs en vogue dans la gastronomie contemporaine, les chefs cuisiniers des restaurants gastronomiques "à la mode" sont aujourd'hui devenus des personnages centraux de la vie culturelle liménienne. Grâce à la forte médiatisation de leurs talents individuels, le métier a conquis un prestige social qui lui était nié auparavant, lorsqu'il était associé aux tâches serviles et féminines.

Cette bienveillance vis-à-vis de l'univers de la gastronomie s'explique, en partie, par l'ascension sociale de la figure du chef cuisinier en Europe et aux États-Unis, montrant ainsi que la réussite sociale passe dorénavant par une réussite économique préalable, et peu importe le domaine. C'est cette possibilité d'affirmation de l'autonomie individuelle, et surtout la possibilité d'être à la tête d'un établissement rentable, qui expliquerait la fin de la situation subordonnée du métier. D'ailleurs, les chefs médiatiques se trouvant actuellement à la tête du boom gastronomique péruvien sont majoritairement issus de milieux sociaux privilégiés. Fils d'hommes politiques, d'hommes d'affaires ou issus de "bonnes familles", leur omniprésence dans les médias est donc révélatrice d'une diversification des sources de prestige social parmi une fraction de Liméniens privilégiés.

La plupart de ces cuisiniers, aujourd'hui âgés de 30 à 42 ans, a intégré des écoles étrangères en raison de l'offre très réduite de ce type de formation à Lima avant la deuxième moitié des années quatre-vingt-dix. Ainsi, Pedro Miguel Schiaffino, pro- 
priétaire du restaurant Malabar, a fait des études à l'American Culinary Institute de New York et à l'Italian Culinary Institute for Foreigners. Gaston Acurio, fondateur d'un véritable empire commercial autour de la gastronomie, est diplômé de l'Escuela de Hosteleria de Madrid et a acquis le statut de chef au Cordon Bleu de Paris. Rafael Piqueras, copropriétaire et chef du restaurant Fusion, a été formé au Cordon Bleu de Lima et à l'Italian Culinary Institute for Foreigners. Rafael Osterling, à la tête de son très chic restaurant Rafael, est diplômé du Cordon Bleu de Londres ; tandis que Jorge Ossio - propriétaire de plusieurs restaurants et cafés - a été formé au Culinary Institute of America ${ }^{(23)}$.

Leur retour au pays entraîna d'importantes transformations dans l'offre gastronomique des dix dernières années. Ils ont apporté non seulement des connaissances et des techniques culinaires nouvelles, mais encore développé un discours gastronomique inédit au Pérou : celui de la "cuisine fusion" à base péruvienne. Influencée par le courant "novo-andin"(24)" et par le courant Fusion $F_{o o d}{ }^{25)}$, la "cuisine fusion" péruvienne est souvent appliquée de manière décomplexée dans les restaurants les plus branchés de la capitale, permettant ainsi aux chefs qui la pratiquent d'affirmer une singularité dite "artistique" et d'asseoir leur hégémonie dans le champ gastronomique ${ }^{(26)}$.

La production de ce nouveau courant gastronomique doit beaucoup à un processus de réappropriation symbolique. Il consiste en grande partie à valoriser un certain nombre d'ingrédients autochtones méconnus des "grandes tables" liméniennes et écartés du monde gastronomique depuis la période coloniale, car ils faisaient partie de la "cuisine d'Indiens". Bien que la cuisine "novo-andine" fut à l'origine de cette dynamique de valorisation, les tenants de la "cuisine" fusion se sont détachés de celle-ci, dans la mesure où ils intègrent aussi dans leurs préparations des éléments amazoniens ${ }^{(27)}$ et des techniques plus novatrices - ce qui leur a aussi permis de mettre en avant leur individualité. Le travail de réappropriation mis en ceuvre par ces cuisiniers consiste, d'abord, à extraire

\section{La production} de ce nouveau courant gastronomique doit beaucoup à un processus de réappropriation symbolique. l'ingrédient de tout contexte préalable neutralisant ainsi une indignité potentielle -, à identifier et à maintenir, ensuite, certaines caractéristiques désirables ou positives de celui-ci et, enfin, à l'assembler avec des éléments issus d'autres plats et d'autres types de cuisine ${ }^{(28)}$. Il vise donc à neutraliser cette sorte "d'exotisme négatif" et à proposer un "exotisme positif", tout en élevant la "cuisine fusion" à base péruvienne au rang d'expression la plus aboutie de la gastronomie du pays. 


\section{Conclusion}

On se trouve ici face à un métissage différent de celui qui a donné naissance à la cuisine criolla liménienne et aux formes culinaires influencées par la migration italienne et asiatique. Ce dernier a été le résultat d'un long processus historique fondé sur d'incessantes confrontations de cultures, faites de rejets et d'appropriations, ayant façonné la construction identitaire d'une société, tandis que la force du nouveau métissage réside principalement dans le discours véhiculé par les producteurs et les agents économiques concernés.

Bien que la "cuisine fusion" à base péruvienne contienne les trois mouvements fondamentaux participant à la recomposition des cultures alimentaires - à savoir la disparition de certains particularismes, l'émergence de nouvelles formes alimentaires et la diffusion à une échelle transculturelle de certains produits et de certaines pratiques alimentaires ${ }^{(29)}$-, sa production s'inscrit principalement dans l'histoire sociale de ses promoteurs et dans leur capacité à diffuser ces "découvertes" auprès d'une clientèle composée par des "pairs". Cependant, le succès international de cette nouvelle gastronomie péruvienne n'aurait certainement pas vu le jour sans la forte articulation des héritages culturels qui ont façonné le métissage culinaire du Pérou.

\section{Notes}

1. En 2006, lors du festival gastronomique Madrid Fusión - le plus grand du monde à ce jour -, la ville de Lima a été désignée "capitale gastronomique de l'Amérique du Sud".

2. Que nous entendons ici comme le résultat d'une confrontation entre une culture coloniale et une culture indigène permettant l'émergence d'une "nouvelle culture". Serge Gruzinski et Louise Bénat-Tachot, Passeurs culturels. Mécanismes de métissage, Paris, MSH, 2001.

3. Parmi les animaux introduits par les Espagnols figurent : les chèvres, les porcs, les lapins, les poules, les oies, les moutons, les pigeons, les vaches. Parmi les céréales et légumes : le riz, les asperges, les olives, le blé, les oignons. Pour ce qui est des fruits, ils ont apporté la canne à sucre, les cerises, les dattes, les abricots et les pêches, les grenades, les figues, les citrons, les melons, les oranges, les poires, les bananes et les vignes. Et, quant aux herbes et condiments, on trouve : le basilic, l'anis, le romarin, entre autres.

4. Un des principaux facteurs qui encouragea l'utilisation de produits locaux par les conquistadors fut la carence d'aliments européens lors des premières années de la conquête, avant leur culture intensive. Par exemple, en période de carence de blé, celui-ci s'est vu remplacé par la farine de maïs, ingrédient qui fut introduit progressivement dans la boulangerie et la pâtisserie. En revanche, la boisson typique des anciens Péruviens - la chicha, préparée à base de maïs fermenté - n'a jamais remplacé le vin. 
5. Le terme "morisque" fait référence à la façon dont les Espagnols appelaient les Maures chassés de l'Espagne, notamment de l'Andalousie, au cours de la Reconquête catholique. Voir François Pouillon, "Simplification ethnique en Afrique du Nord: Maures, Berbères et Arabes (XVIII'-XX' siècles)", Cahiers d'études africaines, n 129, 1993, p. 45.

6. Rosario Olivas Weston, La cocina en el virreinato del Perú, Lima, Universidad de Lima, 1996.

7. Juan José Vega, "La influencia morisca y mora : tres casos especificos", in Olivas Weston Rosario, Cultura, identidad y cocina en el Perú, Lima, USMP, 1996, pp. 157-168.

8. Une autre raison du déclin de l'agriculture préhispanique fut l'introduction d'animaux de pré, tels les ruminants et les moutons, qui arrachent l'herbe et rendent inutilisable le terrain en vue d'une culture postérieure sur le même sol, tandis que les animaux autochtones, tels le lama et l'alpaga, mangent uniquement les feuilles vertes des pacages qui, elles, peuvent repousser. Le délaissement des terres agricoles entraîna une mortalité très élevée de camélidés américains, animaux auxquels les Espagnols n'accordaient aucune importance car ils n'appréciaient pas leur viande en tant qu'aliment.

9. Rodolfo Hinostroza, Primicias de cocina peruana, León, Everest, 2006.

10. On entendra par cuisine criolla toutes les expressions culinaires d'origine côtière, mais ayant été développées principalement dans la capitale péruvienne depuis la période coloniale et jusqu'à l'arrivée des premières migrations andines - au cours de la deuxième décennie du XX⿳⺈ siècle -, indépendamment de l'origine de leurs ingrédients.

Sont ainsi exclues de cette catégorie les cuisines dites "régionales" (andine, amazonienne) - même si elles contiennent des traces de "métissage".

11. Margarita Guerra, La ocupación de Lima. Aspectos económicos, Lima, PUCP, 1996.

12. Mariella Balbi, Los chifas en el Perú. Historia y recetas, Lima, USMP, 1999.

13. Isabelle Lausent-Herrera, "Los inmigrantes chinos en la Amazonia Peruana", Bulletin de l'Institut français d'études andines, XV, n³-4, 1986, pp. 49-60.

14. Lieu de restauration plus proche de la table d'hôte et des cantines que du restaurant, en raison d'une offre limitée de plats et de la faible importance accordée au service.

15. Déformation linguistique du vocable "chifan", qui signifie "manger du riz" en cantonais.

16. Elles concernent notamment certains chifas ayant acquis une très bonne réputation durant la première moitié du siècle dernier et qui, pour des raisons commerciales, n'ont pas intérêt à changer cette appellation.

17. Giovanni Bonfiglio, "La influencia italiana en la cultura culinaria peruana", in Olivas Weston, op. cit.

18. Terme utilisé jusqu'à la fin du XIX e siècle pour désigner un établissement qui commercialisait des produits d'usage quotidien.

19. Assiette froide de poisson blanc cru coupé en petits cubes, mariné dans du jus de citron et mélangé avec de fines bandes d'oignon rouge cru, du sel et du poivre. Plat criollo et "côtier" par excellence, il confortait aussi une dimension identitaire : il représentait l'intégration de la côte à la capitale et à sa modernité.

20. Terme qui signifie "première génération" et qui désigne les fils de Japonais nés au Pérou.

21. Il y a une dizaine d'années, il était toujours possible de retrouver du cebiche mariné pendant des heures dans les rayons "traiteur" de certains supermarchés. De nos jours, cette pratique est tombée en désuétude.

22. Amelia Morimoto, "Presencia nikkei en la cocina peruana", in Olivas Weston Rosario, Cultura, identidad y cocina en el Perú, Lima, USMP, 1996, pp. 257-270.

23. Mirko Lauer et Vera Lauer, La revolución gastronómica del Perú, Lima, USMP, 2006.

24. Courant conçu au cours des années quatre-vingt et commercialisé notamment durant les années quatre-vingt-dix. Il est voué à la récupération d'ingrédients d'origine, comme la kiwicha, le quinoa ou la viande d'alpaga.

25. Terme générique utilisé pour désigner la combinaison de diverses formes de cuisine et de traditions culinaires qui n'étaient pas destinées à être "unies" ou "mariées".

26. Raúl Matta, Enjeux sociaux d'une consommation "haut de gamme". Étude sur les logiques marchandes et sociales au ccur de deux expressions culturelles dans la ville de Lima : l'expérience gastronomique et les fêtes de musique électronique, thèse de doctorat en sociologie, sous la dir. de Marie-France Prévôt-Schapira, université Paris 3-Sorbonne Nouvelle, IHEAL/CREDAL, 2009.

27. Certains d'entre eux parcourent le pays de manière systématique à la recherche de produits méconnus et susceptibles d'intégrer cette gastronomie à la fois inédite et raffinée.

28. Pour regarder quelques exemples à ce sujet, cf. Matta, op. cit, chapitre 5 .

29. Jean-Pierre Poulain, Sociologies de l'alimentation, Paris, PUF, 2005. 